\title{
Central pancreatectomy for a pancreatic metastasis of colon cancer - a case report
}

\author{
Traian Dumitrașcu*, Andra Scarlat*, Silviu Ciurea, Irinel Popescu
}

"Dan Setlacec" Center of General Surgery and Liver Transplantation, Fundeni Clinical Institute, Bucharest, Romania

\section{ABSTRACT}

Background: Isolated pancreatic metastases of other neoplasms are an uncommon pathology, particularly with a colo-rectal cancer origin. Surgery represents the single hope for a long-term survival in these patients, but most of the previously reported patients in the literature underwent standard pancreatic resections. A central pancreatectomy is a non-standardized pancreatic resection, which was exceptionally reported for a malignant pathology.

Case presentation: A 30-years old male, with previously left hemicolectomy for a sigmoid colon adenocarcinoma and left hemi-hepatectomy for liver metastasis, developed an isolated pancreatic isthmus metastasis, which was treated with a central pancreatectomy.

Results: The early postoperative outcome was complicated by a splenic infarction, which required splenectomy. A margins negative resection was observed at the final pathology examination. The patient died with peritoneal recurrence 28 months after the pancreatic resection

Conclusion: A central pancreatectomy appears to be an oncologically safe surgical procedure for isolated pancreatic metastases of other neoplasms, in selected patients.

Key words: central pancreatectomy, pancreatic metastases, prognosis

\section{INTRODUCTION}

A central pancreatectomy is a pancreas-preserving surgical procedure, which was introduced in clinical practice as alternative to distal pancreatectomy, for benign and low-grade malignant pancreatic pathology(1), particularly for the young patients (2).

Endocrine tumors, intraductal papillary mucinous neoplasms and serous/ mucinous cystadenomas located in the pancreatic isthmus and/ or body are the main reported indications for a central pancreatectomy (1). In Fundeni Clinical Institute experience with central pancreatectomies, the serous/ mucinous cystadenomas and the endocrine pancreatic tumors represented more than $75 \%$ of the indications (3).

Although a ductal adenocarcinoma of the pancreas is widely considered an absolute contraindication for a central pancreatectomy (4), however, the

\author{
Corresponding author: \\ Traian Dumitrascu, MD, \\ "Dan Setlacec" Center of General \\ Surgery and Liver Transplantation, \\ Fundeni Clinical Institute, Fundeni \\ Street no 258, Bucharest, Romania, \\ E-mail: traian.dumitrascu76@gmail.com \\ *These authors should be considered \\ as co-first authors
}


oncological safety of this procedure was previously demonstrated for some special pancreatic malignancies, such as pancreatoblastoma (5), and pancreatic metastases of other neoplasms (6). However, a very limited number of patients with parenchyma-sparing resections for pancreatic metastases of other neoplasms were previously reported (3).

Hereby, it is presented the case of a 30 -years old male with a previously resected colon adenocarcinoma which, furthermore, developed an isolated metastasis to the pancreatic isthmus. The surgical management and outcomes of this patient are discussed.

\section{CASE REPORT}

A 30-years old male, with a previously left hemicolectomy for a sigmoid colon adenocarcinoma (T2, N1, MO) in 1997 (with an adjuvant chemotherapy - Mayo protocol), developed in 2004 a single left liver lobe metastasis, invading the biliary convergence, with secondary jaundice. The patient underwent an extrahepatic bile duct resection, en bloc with a left hemihepatectomy and caudate lobe resection, with an uneventful postoperative outcome.

One year after the second surgical procedure (2005), the patient was discovered at the resonance magnetic imaging with a $2 \mathrm{~cm}$ nodular, solid mass located at the level of the pancreatic isthmus (figure 1). No local invasion was observed, as well as any enlarged loco-regional lymph nodes or distant metastases.

Thus, with the preoperative diagnosis of isolated pancreatic metastasis, the patient underwent a central pancreatectomy.

The Fundeni Clinical Institute technique for a central pancreatectomy was previously described for both the open $(7,8)$ and the minimally-invasive (robotic) approach (9).

In the reported patient an open approach was performed, the proximal pancreatic stump was handsewn sutured with interrupted sutures, while the distal pancreatic stump was treated with a duct-to-mucosa anastomosis, with a Roux en $Y$ jejunal loop.

The early postoperative outcome was complicated with a splenic infarction and splenic abscess; a splenectomy was performed on the 12th postoperative day. After the reintervention, the postoperative outcome was uneventful. No pancreatic exocrine or endocrine insufficiencies were detected on the long-term postoperative follow-up.

The final pathology examination revealed a $2 / 1.5$ $\mathrm{cm}$ metastasis to the pancreas of colon cancer origin (adenocarcinoma), with negative resection margins (the length of resected pancreas was $4 \mathrm{~cm}$ ) (figure 2). The examination of the harvested lymph nodes at the inferior and superior margins of the operative specimen did not find any metastases. However, no standard lymph nodes dissection was performed.

The patient refused any adjuvant treatment, and 18 months after the pancreatic resection (2007) was readmitted for upper digestive stenosis. The patient was surgically explored, and peritoneal carcinomatosis was revealed. A palliative gastro-enterostomy was
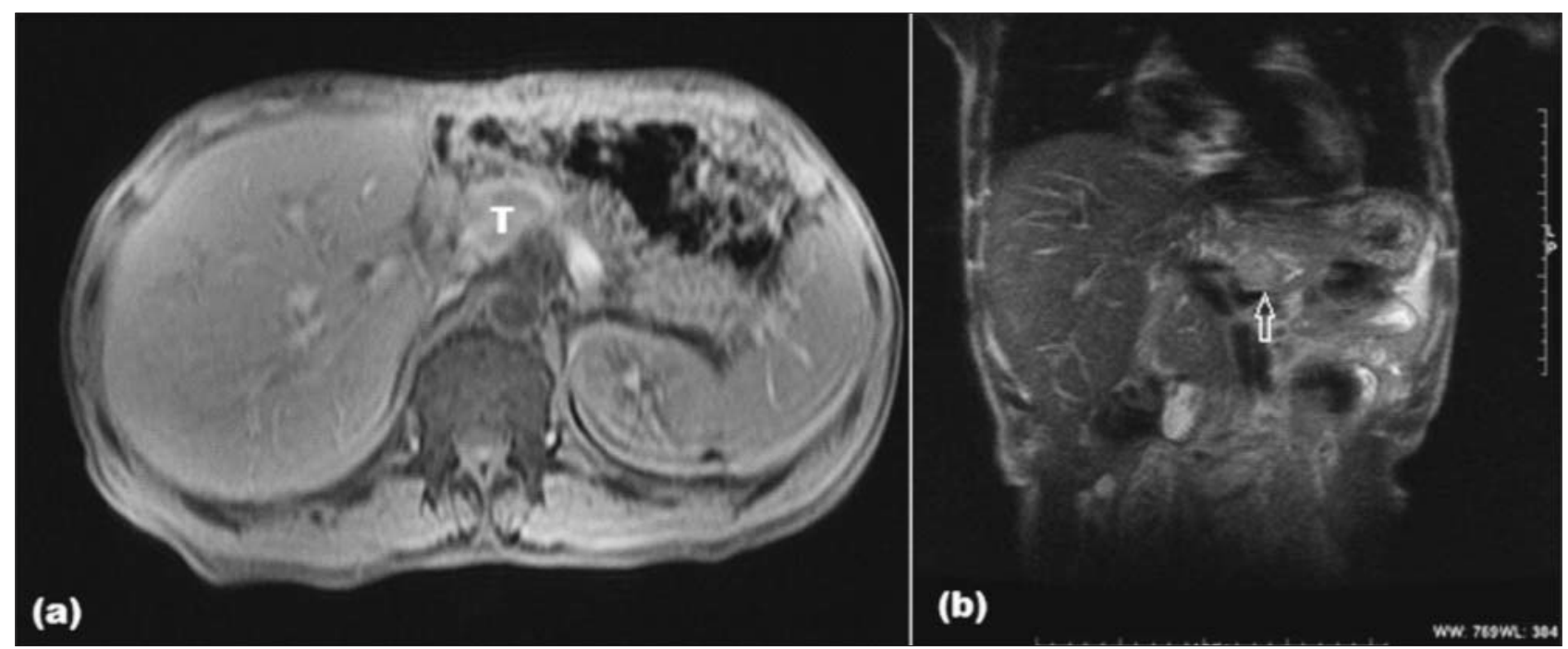

Figure 1 - Isolated pancreatic isthmus metastasis of colon cancer origin - magnetic resonance imaging aspects: (a) coronal plane (T the tumor); (b) frontal plane (the arrow marks the tumor) 


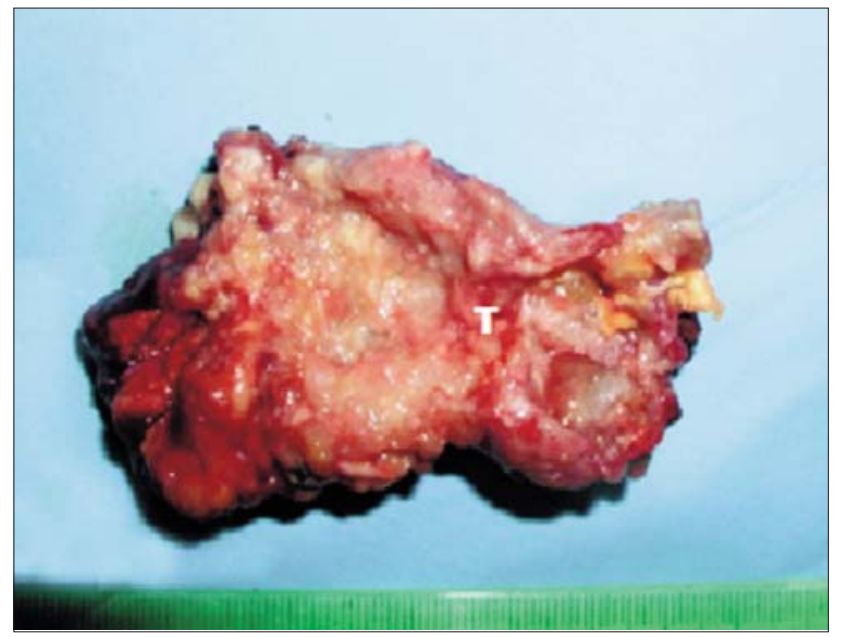

Figure 2 - Central pancreatectomy for an isolated pancreatic isthmus metastasis of colon cancer origin - operative specimen ( $\mathrm{T}$ - the tumor)

performed, with an uneventful postoperative outcome. The patient died 10 month later after the late surgical intervention.

Thus, the patient survived 28 months after the resection of the pancreatic metastasis, with an overall survival of more than 10 years after the resection of the primary colon cancer.

\section{DISCUSSION}

Isolated pancreatic metastases of other neoplasms are an uncommon pathology, representing less than $5 \%$ of all the pancreatic malignant pathology(10;11). A renal cancer represents the main source for pancreatic metastases $(10,11)$.

A review published in 2014 revealed only 25 patients with pancreatic resections for isolated pancreatic metastases of colo-rectal cancer origin, representing $6.2 \%$ of all the reported pancreatic resections for isolated metastases of other neoplasms (10). It appears that a colon cancer very rarely metastasize to the pancreas, as a recent French-population based epidemiological study has shown (12). Nevertheless, a Dutch study has shown no metastases to the pancreas on the long-term follow-up in 5671 patients resected for a colo-rectal cancer (13).

Surgery (i.e., resection) is considered the single hope for a long-term survival in patients with isolated pancreatic metastases of other neoplasms $(11,14)$.

For isolated pancreatic metastases of other neoplasms a routine systematic loco-regional lymph nodes dissection is poorly documented in the literature $(10,11)$. Thus, some surgical teams suggest that a pancreas-preserving resection is oncologicaly safe for pancreatic metastases of other neoplasms $(6,11)$, while others consider only standard pancreatic resections with systematic loco-regional lymph nodes dissection for such a pathology (15).

In a series of patients with standard pancreatic resections (i.e., pancreaticoduodenectomies and distal pancreatectomies) for isolated pancreatic metastases of colo-rectal cancer origin, the survival time of the patients varied between 10 months and 33 months (a median survival time of 17 months)(16). The survival time of the reported patient was 28 months, with a non-standardized pancreatic resection and no standard lymph nodes dissection.

A central pancreatectomy has the potential to better preserve the postoperative pancreatic endocrine and exocrine functions, compared with distal pancreatectomies (1), even if the spleen is preserved during the distal pancreatectomy (17). However, whenever a central pancreatectomy is considered, it would be wise to take into consideration not only the potential benefits, but also its high morbidity, particularly the relatively high rate of complications requiring surgical re-exploration $(3,4,18)$.

\section{CONCLUSION}

A central pancreatectomy appears to be an oncologically safe surgical procedure for isolated pancreatic metastases of other neoplasms, in selected patients. The benefits of a central pancreatectomy, as well as its high morbidity should be discussed with the patient, and the final decision should be taken on an individual based analysis.

\section{Acknowledgement}

This work received financial support through the project entitled "CERO - Career profile: Romanian Researcher", grant number POSDRU/159/1.5/S/135760, cofinanced by the European Social Fund for Sectoral Operational Programme Human Resources Development 2007-2013.

\section{REFERENCES}

1. Iacono C, Verlato G, Ruzzenente A, Campagnaro T, Bacchelli C, Valdegamberi $A$, et al. Systematic review of central pancreatectomy and meta-analysis of central versus distal pancreatectomy. Br J Surg 2013;100(7):873-85.

2. Dumitrascu T, Dima S. [Central pancreatectomy-an alternative for surgical approach of the benign and low-malignant tumors of the pancreatic body in young patients]. Chirurgia (Bucur) 2011;106(5):683-4.

3. Dumitrascu T. [Central pancreatectomy]. PhD Thesis, "Carol Davila" University of Medicine and Pharmacy, Bucharest, 2015.

4. Goudard Y, Gaujoux S, Dokmak S, Cros J, Couvelard A, Palazzo M, et al. Reappraisal of central pancreatectomy a 12-year single-center experience. 
JAMA Surg 2014;149(4):356-63.

5. Dumitrascu T, Stanciulea 0, Herlea V, Tomulescu V, Ionescu M. Central pancreatectomy for pancreatoblastoma in a 16-year-old girl. J Pediatr Surg 2011;46(8):e17-e21.

6. Dumitrascu T, Dima S, Popescu C, Gheonea DI, Ciurea T, Saftoiu A, et al. An unusual indication for central pancreatectomy-late pancreatic metastasis of ocular malignant melanoma. Chirurgia (Bucur) 2008; 103(4):479-85.

7. Ionescu M, Dumitrascu T, Stroescu C, Ciurea S, Pietrareanu D, Mitulescu $\mathrm{G}$, et al. [Central pancreatectomy-indications, technique, outcomes]. Chirurgia (Bucur) 2005:100(5):429-35.

8. Popescu I. Central pancreatectomy. In: Khatri VJ, editor. Atlas of Advanced Operative Surgery.Philadelphia: Elsevier; 2013. p. 232-9.

9. Stanciulea O, Dima S, Eftimie M, Mosteanu I, Popescu I. Central pancreatectomy - a suitable candidate for robotic surgery. J Transl Med Res 2015; 1(20):32-7.

10. Adler H, Redmond CE, Heneghan HM, Swan N, Maguire D, Traynor O, et al. Pancreatectomy for metastatic disease: a systematic review. Eur J Surg Oncol 2014;40(4):379-86.

11. Reddy $\mathrm{S}$, Wolfgang $\mathrm{CL}$. The role of surgery in the management of isolated metastases to the pancreas. Lancet Oncol 2009;10(3):287-93.

12. Ghiringhelli $F$, Hennequin A, Drouillard A, Lepage C, Faivre J, Bouvier AM.
Epidemiology and prognosis of synchronous and metachronous colon cancer metastases: a French population-based study. Dig Liver Dis 2014;46(9):854-8.

13. van Gestel YR, de Hingh IH, van Herk-Sukel MP, van Erning FN, Beerepoot LV, Wijsman JH, et al. Patterns of metachronous metastases after curative treatment of colorectal cancer. Cancer Epidemiol 2014; 38(4):448-54.

14. Strobel $\mathrm{O}$, Hackert T, Hartwig W, Bergmann F, Hinz U, Wente MN, et al. Survival data justifies resection for pancreatic metastases. Ann Surg Oncol 2009;16(12):3340-9.

15. Bassi C, Butturini G, Falconi M, Sargenti M, Mantovani W, Pederzoli P. High recurrence rate after atypical resection for pancreatic metastases from renal cell carcinoma. Br J Surg 2003;90(5):555-9.

16. Sperti C, Pasquali C, Berselli M, Frison L, Vicario G, Pedrazzoli S. Metastasis to the pancreas from colorectal cancer: is there a place for pancreatic resection? Dis Colon Rectum 2009;52(6):1154-9.

17. Dumitrascu T, Scarlat A, Ionescu M, Popescu I. Central pancreatectomy versus spleen-preserving distal pancreatectomy: a comparative analysis of early and late postoperative outcomes. Dig Surg 2012;29(5):400-7.

18. Dumitrascu T, Barbu ST, Purnichescu-Purtan R, Ionescu M, Popescu I. Risk factors for surgical complications after central pancreatectomy. Hepatogastroenterology 2012;59(114):592-8. 